\title{
A SIMILARITY MEASURE FOR CATEGORIZING THE DEVELOPERS PROFILE IN A SOFTWARE PROCESS
}

\author{
Hamid Khemissa ${ }^{1}$, Mohamed Ahmed-nacer ${ }^{2}$ and \\ Abdelkader Belkhir ${ }^{3}$ \\ Computer Systems Laboratory, Computer Science Institute, USTHB \\ University, \\ EL ALIA BP N³2, BAB EZZOUAR ALGERIA. TEL/FAX (00)213 21247917 \\ ${ }^{1}$ hkhemissa@usthb.dz, ${ }^{2}$ anacerecerist.dz and \\ ${ }^{3}$ kaderbelkhirahotmail. com
}

\begin{abstract}
Software development processes need to have an integrated environment that fulfills specific developer needs. In this context, this paper describes the modeling approach SAGM ((Similarity for Adaptive Guidance Model) that provides adaptive recursive guidance for software processes, and specifically tailored regarding the profile of developers. A profile is defined from a model of developers, through their roles, their qualifications, and through the relationships between the context of the current activity and the model of the activities. This approach presents a similarity measure that evaluates the similarities between the profiles created from the model of developers and those of the development team involved in the execution of a software process. This is to identify the profiles classification and to deduce the appropriate type of assistance (that can be corrective, constructive or specific) to developers.
\end{abstract}

\section{KEYWORDS}

Software process modeling, Process engineering, Adaptive guidance profile, Similarity measure.

\section{INTRODUCTION}

Improving quality and productivity of software development requires assisting developers at both methodology level and consistency results level [1]. A guidance model in software engineering should combine the needed features to build the support system $[2,3]$.

Several PSEEs (Process-Centered Software Engineering Environments) [2, 4] deal the assistance aspect in the support of the software product development. Some PSEEs use an assistance description structured in steps like prescribing systems or proactive systems to control the operations carried out by the developer. The main limitations of these PSEEs are:

The human actor has a central role in the progress of the development process regardless of his profile (qualifications and behavior). 
$>$ The basic guidance is defined as a global orientation core whatever the profiles of both the activity and the developer.

The selection of the appropriate type of guidance is often more intuitive and not suitable.

To respond to these limits, several studies $[2,3,5,6]$ try to offer more flexibility in the language of software process modeling and a more adapted base of support and control. This tendency aims to define interventions of direct and adaptive assistance during the software process progress [7]. The following PSEEs included in the M1 level are as:

ADELE/APEL is based on a reactive database. It proposes a global assistance of proscriptive type and automates part of the development process using triggers $[8,9]$.

RHODES/PBOOL+ uses an explicit description of a development process. The software processes are modeled in PBOOL language [10]. The activities are associated to a guidance system with various scenarios of possible realization.

ADDD/ALADYN provides process automation and control the impact in a concrete system. The task hierarchy is used to organize the process descriptions, called policies. Several aspects are grouped and treated in a policy. A policy can be instantiated for several tasks. The instantiated triggers are rules of the form event-condition-action (ECA) and used to implement a reactive behavior [11].

On the M2 level of Meta model, SPEM [12] introduced the concept of "Guidance" in the "Managed Content" package by defining the stereotype "Guidance". According to SPEM," the Guidance is a describable element which provides additional information to define the describable elements of a modeling. It also offers, through the stereotype "Guidance_kind" different types of guidance such as: Template, Guidelines, Checklists, etc. ..

However, the selection of guidance types remains defined in a manual and in an intuitive way. It depends on the experience and on the informal personality of the project manager. In addition, the proposed guidance is not adaptive to the actor's profile (role, qualifications and behavior).

In considering the principal limitations of PSEEs and essential characteristics of our approach as the context adaptation aspect and the abstraction levels, a comparative table of the studied Meta models is as follows:

Table 1. A Comparative table of the studied Meta models

\begin{tabular}{|c|c|c|c|c|}
\hline Meta model & ADELE /APEL & $\begin{array}{c}\text { RHODES / } \\
\text { PBOOL+ }\end{array}$ & ADDD / ALADYN & SPEM \\
\hline $\begin{array}{c}\text { Global guidance } \\
\text { core }\end{array}$ & Global & Global & $\begin{array}{c}\text { Customized for } \\
\text { each task }\end{array}$ & Global \\
\hline $\begin{array}{c}\text { Human performer } \\
\text { profile oriented } \\
\text { guidance }\end{array}$ & Not adapted & $\begin{array}{c}\text { Nonsidered } \\
\text { strategy Model }\end{array}$ & Adapted & Not adapted \\
\hline $\begin{array}{c}\text { Context } \\
\text { Guidance }\end{array}$ & Not adapted & Adapted & Not invoked & Intuitive \\
\hline selection
\end{tabular}


Computer Science \& Information Technology (CS \& IT)

\begin{tabular}{|c|c|c|c|c|}
\hline $\begin{array}{c}\text { Explicit activity } \\
\text { abstraction }\end{array}$ & $\begin{array}{c}\text { Explicit } \\
\text { abstraction }\end{array}$ & $\begin{array}{c}\text { Implicit } \\
\text { abstraction }\end{array}$ & Implicit abstraction & $\begin{array}{c}\text { Explicit } \\
\text { abstraction }\end{array}$ \\
\hline $\begin{array}{c}\text { Explicit task } \\
\text { abstraction }\end{array}$ & $\begin{array}{c}\text { Implicit } \\
\text { abstraction }\end{array}$ & Not invoked & Explicit abstraction & $\begin{array}{c}\text { Explicit } \\
\text { abstraction }\end{array}$ \\
\hline $\begin{array}{c}\text { Process Modeling } \\
\text { Language(PML) }\end{array}$ & $\begin{array}{c}\text { WPEL } \\
\text { Writh predefined } \\
\text { primitives }\end{array}$ & $\begin{array}{c}\text { With explicit } \\
\text { primitives }\end{array}$ & $\begin{array}{c}\text { ALADYN } \\
\text { Not explicitly } \\
\text { mentioned }\end{array}$ & $\begin{array}{c}\text { UML Profile } \\
\text { primitive }\end{array}$ \\
\hline
\end{tabular}

The current tendency is that developers would like to have integrated environments that are suitable to specific needs according to the role and the characteristics of each developer and closed to the context of the underway task. However, the provided efforts to develop such environments remain an insufficient contribution.

In this context, our conceptual model is based on the conventional reasoning of software processes enriched by the "Adaptive Guidance" element which supervises the running of the activities. It also provides adaptive support to the actor. It is described by the following figure:

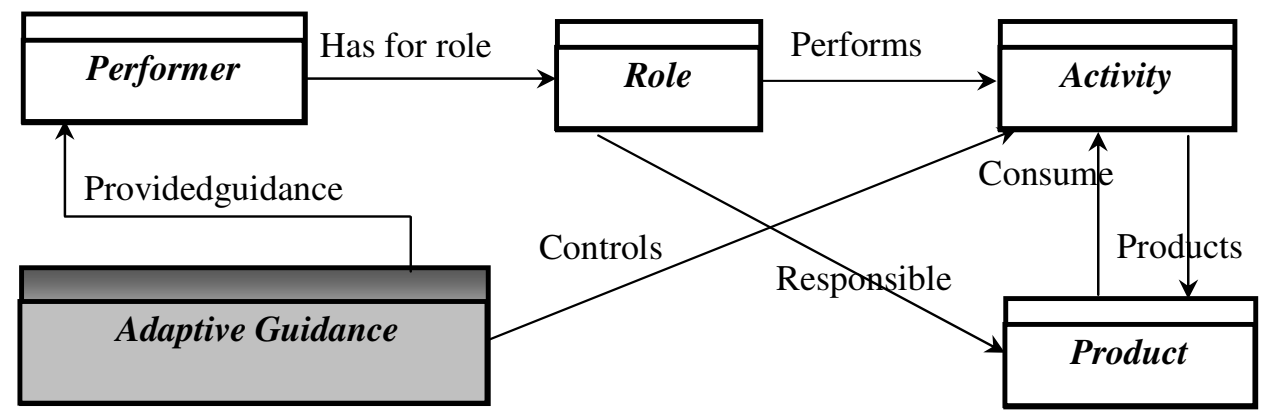

Figure 1. : Conceptual Model with Adaptive Guidance

This tendency of adaptive guidance environments is yet a subject of much research focusing on defining the concepts and objectives of process modeling software-based adaptive guidance $[2,3$, $6]$.

For the sake of productivity and development time, our goal is to establish an optimal relationship between profile of guidance type and the adapted developer's profile to the context of development. The context is defined by the activity model, the developer and team development $[2,3,13]$. It is interesting to have an operator to assess the similarities within the handled data; this operator is the similarity function $[13,14,15,16]$. The numerical similarity measures turn out to be extremely flexible employment. They are able to work on a broad spectrum of data types and it is fairly easy to introduce into the calculation, (statistical approximations if the underlying information is complex). In addition, similarities quantification by a continuous value implies that it is always possible and easy to compare pairs of objects. This is not the case for the symbolic similarity; treatment of numerical values is often done in an unsatisfactory way by rewriting these values in symbolic form $[14,16]$.

Our approach operates in the optimization of profiles classes in relation to the semantics of data manipulation. It defines a system for processing the similarity index and classification of guidance profiles. For this, we have to design a classifier in order to facilitate the analysis of our 
population and the type of assistance offered to appropriate developers involved in a software process.

The second section summarizes the technique of assessing similarity. Section three presents our approach (to model similarity with the software process), in addition to the implementation and practical evaluation of our approach by giving algorithms and related results. The last section concludes and presents future works perspectives.

\section{SIMILARITY MEASURE}

A similarity measure is defined on the set $\mathrm{N}$ (developers, documents, websites ...). Each object is described by $\mathrm{m}$ features. Each feature can be present or absent in every object. A measure of similarity, denoted by s, (between the elements of $\mathrm{N}$ is a specific application of $\mathrm{NxN}$ in $\mathrm{R}$ and satisfying some properties $[14,15])$.

Examples of the use of similarity techniques are described in cases of heterogeneous binary data [17]. To transform a direct measure of similarity s into a dissimilarity measure $\mathrm{d}$, we can apply the following formula: $\quad d(x, y)=\operatorname{smax}-s(x, y)$.

Thus, each element $\mathrm{x}$ is associated to a binary vector $\left(\mathrm{x}_{1}, \mathrm{x}_{2}, \ldots, \mathrm{x}_{\mathrm{m}}\right)$ such that:

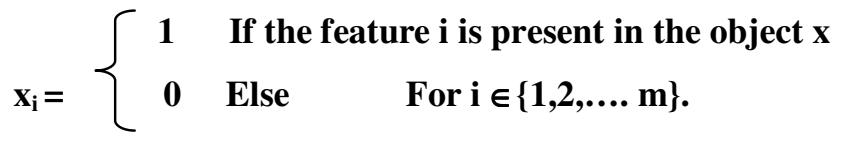

The $m$ characteristics are considered of equal importance and each object has at least one feature present. Note by:

- a: The number of common characteristics between $x$ and $y$.

- b: The number of features present in $x$ but not $y$.

- c: The number of features present in $y$ but not $x$.

d: The number of missing features in $x$ and $y$.

Thus, the similarity measure $\mathrm{s}$ is given by the following formula:

$$
\forall \mathbf{x}, \mathbf{y} \in \mathbf{N}: \mathbf{s}(\mathbf{x}, \mathbf{y})=\frac{2 a+b+c}{2(\mathrm{a}+\mathrm{b}+\mathrm{c})}
$$

We can deduce the measure of dissimilarity from the following formula:

$$
\forall \mathbf{x}, \mathbf{y} \in \mathbf{N}: \mathbf{d}(\mathbf{x}, \mathbf{y})=\mathbf{l}-\frac{2 a+b+c}{2(a+b+c)}=\frac{b+c}{2(a+b+c)}
$$

Besides this general form, there are also other forms of similarity measures such as binary data $[14,15,17]$ as those given in table 2 . 
Table 2. Different forms of similarity measures

\begin{tabular}{|c|c|}
\hline Author & Definition \\
\hline Russel, Rao & $\mathrm{S}=\frac{a}{\mathrm{~m}}$ \\
\hline Kendall, Sokal-Michener & $\mathrm{S}=\frac{a+d}{\mathrm{~m}}$ \\
\hline Rogers, Tanimoto & $\mathrm{S}=\frac{a+d}{\mathrm{~m}+\mathrm{b}+\mathrm{c}}$ \\
\hline Hamann & $\mathrm{S}=\frac{a+d-b-c}{\mathrm{~m}}$ \\
\hline Sokal, Sneath & $\mathrm{S}=\frac{b+c}{\mathrm{a}+\mathrm{d}}$ \\
\hline
\end{tabular}

To review and evaluate the effect of binary similarity measures, we will illustrate all the similarity measures in an example as follows: Consider a set of 16 objects (A1, A2, . , A16), each object has thirteen (13) features present or absent $(\mathrm{C} 1, \mathrm{C} 2, \ldots, \mathrm{C} 13)$, all these data are illustrated in the following table:

Table 3. Different forms of similarity measures

\begin{tabular}{|c|c|c|c|c|c|c|c|c|c|c|c|c|c|}
\hline & $\mathrm{C}_{1}$ & $\mathrm{C}_{2}$ & $\mathrm{C}_{3}$ & $\mathrm{C}_{4}$ & $\mathrm{C}_{5}$ & $\mathrm{C}_{6}$ & $\mathrm{C}_{7}$ & $\mathrm{C}_{8}$ & $\mathrm{C}_{9}$ & $\mathrm{C}_{10}$ & $\mathrm{C}_{11}$ & $\mathrm{C}_{12}$ & $\mathrm{C}_{13}$ \\
\hline $\mathrm{A}_{1}$ & $\mathbf{1}$ & $\mathbf{0}$ & $\mathbf{0}$ & $\mathbf{1}$ & $\mathbf{0}$ & $\mathbf{0}$ & $\mathbf{0}$ & $\mathbf{0}$ & $\mathbf{1}$ & $\mathbf{0}$ & $\mathbf{0}$ & $\mathbf{1}$ & $\mathbf{0}$ \\
\hline $\mathrm{A}_{2}$ & $\mathbf{1}$ & $\mathbf{0}$ & $\mathbf{0}$ & $\mathbf{1}$ & $\mathbf{0}$ & $\mathbf{0}$ & $\mathbf{0}$ & $\mathbf{0}$ & $\mathbf{1}$ & $\mathbf{0}$ & $\mathbf{0}$ & $\mathbf{0}$ & $\mathbf{0}$ \\
\hline $\mathrm{A}_{3}$ & $\mathbf{1}$ & $\mathbf{0}$ & $\mathbf{0}$ & $\mathbf{1}$ & $\mathbf{0}$ & $\mathbf{0}$ & $\mathbf{0}$ & $\mathbf{0}$ & $\mathbf{1}$ & $\mathbf{0}$ & $\mathbf{0}$ & $\mathbf{0}$ & $\mathbf{1}$ \\
\hline $\mathrm{A}_{4}$ & $\mathbf{1}$ & $\mathbf{0}$ & $\mathbf{0}$ & $\mathbf{1}$ & $\mathbf{0}$ & $\mathbf{0}$ & $\mathbf{0}$ & $\mathbf{0}$ & $\mathbf{1}$ & $\mathbf{0}$ & $\mathbf{0}$ & $\mathbf{1}$ & $\mathbf{1}$ \\
\hline $\mathrm{A}_{5}$ & $\mathbf{1}$ & $\mathbf{0}$ & $\mathbf{0}$ & $\mathbf{1}$ & $\mathbf{0}$ & $\mathbf{0}$ & $\mathbf{0}$ & $\mathbf{0}$ & $\mathbf{1}$ & $\mathbf{1}$ & $\mathbf{0}$ & $\mathbf{1}$ & $\mathbf{0}$ \\
\hline $\mathrm{A}_{6}$ & $\mathbf{1}$ & $\mathbf{0}$ & $\mathbf{0}$ & $\mathbf{1}$ & $\mathbf{0}$ & $\mathbf{0}$ & $\mathbf{0}$ & $\mathbf{0}$ & $\mathbf{1}$ & $\mathbf{1}$ & $\mathbf{0}$ & $\mathbf{1}$ & $\mathbf{0}$ \\
\hline $\mathrm{A}_{7}$ & $\mathbf{0}$ & $\mathbf{1}$ & $\mathbf{0}$ & $\mathbf{1}$ & $\mathbf{0}$ & $\mathbf{0}$ & $\mathbf{0}$ & $\mathbf{0}$ & $\mathbf{1}$ & $\mathbf{1}$ & $\mathbf{0}$ & $\mathbf{1}$ & $\mathbf{0}$ \\
\hline $\mathrm{A}_{8}$ & $\mathbf{0}$ & $\mathbf{1}$ & $\mathbf{0}$ & $\mathbf{0}$ & $\mathbf{1}$ & $\mathbf{1}$ & $\mathbf{0}$ & $\mathbf{0}$ & $\mathbf{0}$ & $\mathbf{1}$ & $\mathbf{0}$ & $\mathbf{0}$ & $\mathbf{0}$ \\
\hline $\mathrm{A}_{9}$ & $\mathbf{0}$ & $\mathbf{1}$ & $\mathbf{0}$ & $\mathbf{0}$ & $\mathbf{1}$ & $\mathbf{1}$ & $\mathbf{0}$ & $\mathbf{0}$ & $\mathbf{0}$ & $\mathbf{0}$ & $\mathbf{1}$ & $\mathbf{0}$ & $\mathbf{0}$ \\
\hline $\mathrm{A}_{10}$ & $\mathbf{0}$ & $\mathbf{1}$ & $\mathbf{0}$ & $\mathbf{0}$ & $\mathbf{1}$ & $\mathbf{1}$ & $\mathbf{0}$ & $\mathbf{1}$ & $\mathbf{0}$ & $\mathbf{1}$ & $\mathbf{1}$ & $\mathbf{0}$ & $\mathbf{0}$ \\
\hline $\mathrm{A}_{11}$ & $\mathbf{1}$ & $\mathbf{0}$ & $\mathbf{0}$ & $\mathbf{0}$ & $\mathbf{1}$ & $\mathbf{1}$ & $\mathbf{0}$ & $\mathbf{0}$ & $\mathbf{0}$ & $\mathbf{1}$ & $\mathbf{0}$ & $\mathbf{0}$ & $\mathbf{0}$ \\
\hline $\mathrm{A}_{12}$ & $\mathbf{0}$ & $\mathbf{0}$ & $\mathbf{1}$ & $\mathbf{0}$ & $\mathbf{1}$ & $\mathbf{1}$ & $\mathbf{0}$ & $\mathbf{0}$ & $\mathbf{0}$ & $\mathbf{1}$ & $\mathbf{1}$ & $\mathbf{0}$ & $\mathbf{0}$ \\
\hline $\mathrm{A}_{13}$ & $\mathbf{0}$ & $\mathbf{0}$ & $\mathbf{1}$ & $\mathbf{0}$ & $\mathbf{1}$ & $\mathbf{1}$ & $\mathbf{0}$ & $\mathbf{1}$ & $\mathbf{0}$ & $\mathbf{1}$ & $\mathbf{1}$ & $\mathbf{0}$ & $\mathbf{0}$ \\
\hline $\mathrm{A}_{14}$ & $\mathbf{0}$ & $\mathbf{0}$ & $\mathbf{1}$ & $\mathbf{0}$ & $\mathbf{1}$ & $\mathbf{1}$ & $\mathbf{1}$ & $\mathbf{1}$ & $\mathbf{0}$ & $\mathbf{0}$ & $\mathbf{1}$ & $\mathbf{0}$ & $\mathbf{0}$ \\
\hline $\mathrm{A}_{15}$ & $\mathbf{0}$ & $\mathbf{0}$ & $\mathbf{1}$ & $\mathbf{0}$ & $\mathbf{1}$ & $\mathbf{1}$ & $\mathbf{1}$ & $\mathbf{1}$ & $\mathbf{0}$ & $\mathbf{0}$ & $\mathbf{1}$ & $\mathbf{0}$ & $\mathbf{0}$ \\
\hline $\mathrm{A}_{16}$ & $\mathbf{0}$ & $\mathbf{0}$ & $\mathbf{1}$ & $\mathbf{0}$ & $\mathbf{1}$ & $\mathbf{1}$ & $\mathbf{1}$ & $\mathbf{0}$ & $\mathbf{0}$ & $\mathbf{0}$ & $\mathbf{0}$ & $\mathbf{0}$ & $\mathbf{0}$ \\
\hline
\end{tabular}

In applying the measures of similarities between some pairs of objects, we obtain a rate of similarity on the similarity function used:

Table 4. A rate of similarity on the similarity function used

\begin{tabular}{|c|c|c|c|c|}
\hline & $\mathbf{S}\left(\mathbf{A}_{1}, \mathbf{A}_{\mathbf{2}}\right)$ & $\mathbf{S}\left(\mathbf{A}_{\mathbf{1}, \mathbf{A 1 2}}\right)$ & $\mathbf{S}\left(\mathbf{A}_{\mathbf{1 2}}, \mathbf{A}_{\mathbf{1 6}}\right)$ & $\mathbf{S}\left(\mathbf{A}_{\mathbf{1}}, \mathbf{A}_{\mathbf{1 6}}\right)$ \\
\hline Russel, Rao & 0.23 & 0.00 & 0.23 & 0.00 \\
\hline $\begin{array}{c}\text { Kendall, Sokal- } \\
\text { Michener }\end{array}$ & 0.92 & 0.31 & 0.77 & 0.38 \\
\hline Rogers, Tanimoto & 0.86 & 0.18 & 0.63 & 0.24 \\
\hline Hamann & 0.85 & -0.38 & 0.54 & -0.23 \\
\hline Sokal, Sneath & 0.96 & 0.47 & 0.87 & 0.56 \\
\hline
\end{tabular}

The previous results give relations that may exist between different data from a sample objects and deduce statistical information for describing more condensed key information contained in these data. We also seek to classify data into different subgroups that are similar. According to 
the area, the nature of this knowledge is different in data analysis; information will be taken into account instead of a statistical nature.

\section{Similarities In THE SOFTWARE PROCESS}

The proposed guidance system [3] addresses multiple views providing assistance to stakeholders. Our approach aims to optimize the profile classes. To be adaptive to both the context and identified needs, our model of adaptive guidance covers two levels of abstraction. It is based on a set of task and activity model, the model developer and development team, as well as the selection criteria specified by the mode of access for responding the objects of support by the defined assistance interventions (Figure 2.). The instantiation of this system is through rules of assistance detailed with the requirements for initiating appropriate actions to support a particular context $[2,3,5]$.

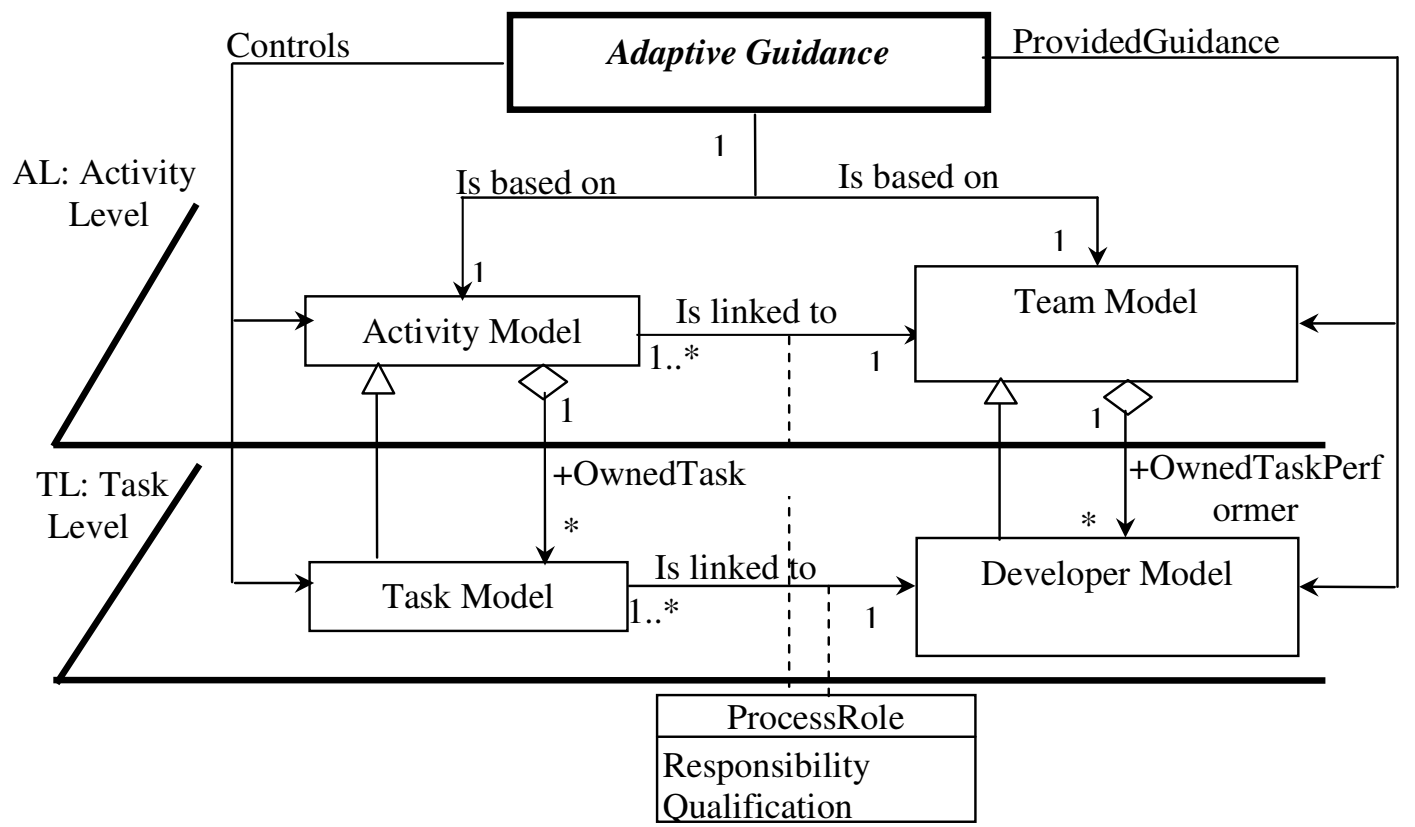

Figure 2. : Adaptive Guidance Model.

\subsection{The Adaptive guidance model}

This assistance system is based on the major models: the activity model, the developer model and the team development model.

a) The activity model: models the workflow, it is defined by:

- A hierarchical list of tasks,

- A mode of progression in the activity ensuring that all tasks can be performed under control in a preset order established by the designer,

- A temporal mode of progression specifying deadlines for completion.

The aspects of the activity model are useful for the assistance system to provide assistance on contextual growth in activity. 
b) The developer model: defines the specific properties of each user. It allows our model to make adaptation according to these properties while maintaining the activity model. These properties can be either static or dynamic.

- The static aspect refers to the user characteristics:

$\circ$ his expertise in the field,

- his familiarity with the software process model or with the software process,

$\circ$ his role in the activity.

- The dynamic aspect refers to the behavior of using the assistance system, the assistance system must be interpreted during the use of the process or system software support, for example :

○ the fact to execute, to define or to complete resource of software process,

$\circ$ the workload of an activity,

$\circ$ his reaction to a message of support.

c) The development team model: development environments allow exchanges and collaborative work. The assistance system can then construct a development team model that represents elements of the team. Example: trace of the various activities of the team as well as different interactions allow the developer to have a script about his own progress in the activity and the progression of the team. The properties of this model can be static or dynamic order.

- The static dimension references skills and team performance in the field of collaboration and distribution of task.

- The dynamic dimension deals with the behavior of the development team. It describes the actions taken by the team during the course of software process.

These data constitute indications that can be interpreted on the use of the assistance by the developer.

\subsection{The assistance intervention}

During the construction or interpretation of a software process model, the proposed model for assistance allows the developer to choose various support functions, namely:

3.2.1. Controlling and taking corrective initiative: protect the user of his own initiatives when they are inappropriate, inadequate initiative under progress.

3.2.2. Controlling and taking constructive initiative: the ability to take positive initiatives, executing and combining the performance of operations without user intervention.

3.2.3. Specific assistance: analyze the impact projection to avoid deadlocks or delays.

\subsection{The profiles categorization}

For the sake of productivity and optimal lead time, we were led to define an effective process for allocating appropriate guidance's. This efficiency is based on the process of maximizing the number of profiles classes to be considered in a development system. We will present our analysis of similarity and classification of our population.

The conception of the processing system will be done through various algorithms. They process both similarity index and hierarchical classification threshold for different profiles. To avoid an important dissemination of similarity, this classification will be ordered by level of similarity 
index. This classification will serve as the basis for the selection and assignment of appropriate types of assistance.

To reach an objective comparison between the profiles, an operator should be used for calculating similarity based on the instantaneous evaluation of selected features and associated weights. Despite the fact that this evaluation is not formal, it remains a crucial step for the classification. For this, we use the notion of symbolic learning for instant evaluation of some attributes of the profile as the behavior of the developer or development team.

\subsubsection{Algorithm for computing Similarity Index}

The evaluation of characteristics is based on the evolution of developer productivity. The weight value of each feature indicates the degree of its importance. The approach used for the evaluation of characteristics is based on "COCOMO II" work $[19,20]$. The table of weights could be refined as soon as we have more data.

Consider two people profiles symbolized by:

$$
\begin{aligned}
& \mathbf{X}=\left(\mathbf{x}_{1}, \mathbf{x}_{2}, \mathbf{x}_{3}, \ldots \ldots \ldots \ldots \ldots, \mathbf{x}_{\mathbf{n}}\right) \\
& \mathbf{Y}=\left(\mathbf{y}_{1}, \mathbf{y}_{2}, \mathbf{y}_{3}, \ldots \ldots \ldots \ldots \ldots . ., \mathbf{y}_{\mathbf{n}}\right)
\end{aligned}
$$

Each characteristic is related to a weight representing its impact in the degree of similarity and symbolized by: $\mathbf{W}_{\mathbf{i}}=\left(\mathbf{w}_{\mathbf{i} 1}, \mathbf{w}_{\mathrm{i} 2}, \mathbf{w}_{\mathrm{i} 3}, \ldots \ldots \ldots \ldots \ldots \ldots . . . . ., \mathbf{w}_{\mathrm{in}}\right)$

Example: a family of two profiles is semantically described in table 4 and table 5 . The semantics evaluation and the weighting are determined by the project manager on an ongoing project $[3,19$, $20]$.

\begin{tabular}{|c|c|c|c|}
\hline & Features & Evaluation of profile 1 & Evaluation of profile 2 \\
\hline \multirow{3}{*}{ 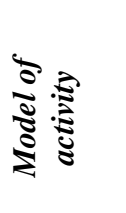 } & Density of tasks in the activity & High & Medium \\
\hline & Complexity level & Medium & Low \\
\hline & Activity Type & Tolerance zero & Margin Free \\
\hline \multirow{4}{*}{ 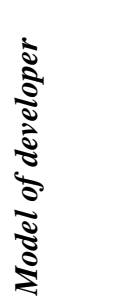 } & Role & Critical & Classic \\
\hline & Competence & High & Medium \\
\hline & $\begin{array}{c}\text { Familiarity with Process } \\
\text { Software }\end{array}$ & Medium & Low \\
\hline & Behavior for assistance & Adequate & Acceptable \\
\hline \multirow{2}{*}{ 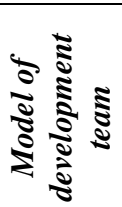 } & Skill Area Collaboration & High & Medium \\
\hline & Behavior for assistance & Acceptable & Adequate \\
\hline
\end{tabular}

Table 5. the profiles evaluation.

To scan the semantics evaluation, we associate the weight corresponding to the consideration according to each attribute. 
Table 6. Table of weights

\begin{tabular}{|c|c|}
\hline $\mathrm{W}[1]$ & $\mathrm{P} 2$ \\
\hline $\mathrm{W}[2]$ & $\mathrm{P} 2$ \\
\hline $\mathrm{W}[3]$ & $\mathrm{P} 3$ \\
\hline $\mathrm{W}[4]$ & $\mathrm{P} 2$ \\
\hline $\mathrm{W}[5]$ & $\mathrm{P} 2$ \\
\hline $\mathrm{W}[6]$ & $\mathrm{P} 2$ \\
\hline $\mathrm{W}[7]$ & $\mathrm{P} 2$ \\
\hline $\mathrm{W}[8]$ & $\mathrm{P} 2$ \\
\hline $\mathrm{W}[9]$ & $\mathrm{P} 4$ \\
\hline
\end{tabular}

With $[i] \in[1,5]$. Where Pi represents the computing value

The algorithm processing is as follows: For any feature, whether it is identical to the profiles, we increment the similarity index by the weight of this feature, otherwise, if the difference between the two characteristics is $<1 / 2$, we add half the weight of it, otherwise, we move to the next feature.

The value $1 / 2$ represents the average distance between two successive levels of an attribute evaluation.

After all iterations, the similarity function obtained is formalized as follows:

$$
S(x, y)=\frac{A(x, y)}{\sum W[i]}
$$

\section{With:}

- $\mathrm{X}$ and $\mathrm{Y}$ represent the characteristics of the two profiles.

- W [ ] represents the weighting of each feature.

- A (x, y) represents the sum of the weights between the two profiles, it is included between 0 and $\Sigma \mathrm{W}[\mathrm{i}]$.

The similarity function developed verifies the properties of a similarity measure.

$\forall x, y$ two profiles $\in N$

$>$ If the profiles $(x, y)$ are identical

Then $A(x, y)=A(x, x)=\sum W[i]$ with $i=1 . .9 \Rightarrow S(x, y)=A(x, y) / \sum W[i]=1$

$>$ If the profiles $(x, y)$ are totally different (the values are all above features $>1 / 2)$. Then for

any characteristic $A(x, y)=0 \Rightarrow S(x, y)=A(x, y) / \sum W[i]=0$.

$>$ If any profiles are neither identical nor different then "It is appropriate to consider three subsets possible through the following 03 cases":

This allowed us to affirm that:

$\checkmark \forall \mathrm{x}, \mathrm{y}$ two profiles $\in \mathrm{N}$, The similarity function $\mathrm{S}(\mathrm{x}, \mathrm{y}) \in[0,1]$.

$\checkmark \forall \mathrm{x}, \mathrm{y}$ two profiles $\in \mathrm{N}$, then $\mathrm{S}(\mathrm{x}, \mathrm{x})=\mathrm{S}(\mathrm{y}, \mathrm{y}) \geq \mathrm{S}(\mathrm{x}, \mathrm{y})$. 
(1) $A(x, y)=0 \quad$ for features with a difference $>1 / 2$

(2) $A(x, y)=\Sigma 1 / 2 W[i]$ for the characteristics with a difference $<1 / 2$

(3) $A(x, y)=\Sigma W[i] \quad$ for completely identical characteristics.

\section{Finally for all characteristics:}

$$
\begin{aligned}
A(x, y) & =A(x, y)_{(1)}+A(x, y)_{(2)}+A(x, y)_{(3)} \\
& =0_{(1)}+1 / 2 \sum W[i]_{(2)}+\sum W[i]_{(3)}
\end{aligned}
$$

Then

$$
S(x, y)=A(x, y) / \sum W[i]=\left(0_{(I)}+1 / 2 \sum W[i]_{(2)}+\sum W[i]_{(3)}\right) /\left(\sum W[i]_{(1)}+\right.
$$

Input: The Profiles of Two Developers $X$ and $Y$. - Table Weights $W[]$. Output: The value of similarity index $S(x, y) \in[0,1]$.

\section{Begin:}

$A(x, y)=0 ; / /$ Similarity index between $X$ and $Y$

For all characteristics $\mathrm{X} i$ and $Y i$ Do

If both characteristics are identical

Then $A(x, y)=A(x, y)+W[i]$;

Else

$$
\text { If }(\mid X i-Y i) \mid<1 / 2)
$$

Then $A(x, y)=A(x, y)+1 / 2 W[i]$

\section{End if;}

\section{End if;}

\section{End.}

Figure 3. : Algorithm for calculating similarity index.

Example: based on the assessing approach of the COCOMO model, the quantification of each characteristic of a profile $\mathrm{P}$ is on the data range] 0,2 [. It is usually done through three steps, described by high, medium or low levels contribution, applying the following rules:

1: impact of middle order.

$<1$ : positive impact.

$>1$ : negative impact. 
Table 7. The profiles evaluation

\begin{tabular}{|c|c|c|c|c|c|}
\hline & Features & profile 1 & profile 2 & profile 3 & profile 4 \\
\hline \multirow{3}{*}{ 苛 } & $\begin{array}{c}\text { Density of tasks in the } \\
\text { activity }\end{array}$ & 1.65 & 1.20 & 1.10 & 1.65 \\
\hline & Complexity level & 1.00 & 0.60 & 1.00 & $\mathbf{1 . 0 0}$ \\
\hline & Activity Type & 1.70 & 1.20 & 1.20 & 1.70 \\
\hline \multirow{4}{*}{ 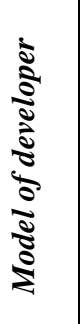 } & Role & 1.15 & 0.70 & 1.15 & 1.60 \\
\hline & Competence & 055 & 1.00 & 1.00 & 0.55 \\
\hline & $\begin{array}{l}\text { Familiarity with } \\
\text { Process Software }\end{array}$ & 0.40 & 0.80 & 0.35 & 0.40 \\
\hline & Behavior for assistance & 0.40 & 0.60 & 0.40 & 0.40 \\
\hline \multirow{2}{*}{ 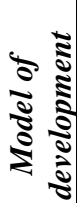 } & $\begin{array}{c}\text { Skill Area } \\
\text { Collaboration }\end{array}$ & 0.70 & 0.70 & 0.70 & 0.70 \\
\hline & $\begin{array}{l}\text { Behavior for } \\
\text { assistance }\end{array}$ & 0.95 & 0.10 & 0.95 & 0.95 \\
\hline
\end{tabular}

The weight value of each feature indicates the degree of its importance. The project manager associates the value correspondence table of weights, for example.

Table 8. Table of weights

\begin{tabular}{|c|c|}
\hline $\mathrm{W}[1]$ & 1 \\
\hline $\mathrm{W}[2]$ & 1 \\
\hline $\mathrm{W}[3]$ & 2 \\
\hline $\mathrm{W}[4]$ & 1 \\
\hline $\mathrm{W}[5]$ & 1 \\
\hline $\mathrm{W}[6]$ & 1 \\
\hline $\mathrm{W}[7]$ & 1 \\
\hline $\mathrm{W}[8]$ & 1 \\
\hline $\mathrm{W}[9]$ & 2 \\
\hline
\end{tabular}

Based on our approach, the calculation of the similarity value between profiles is given by:

Table 9. The similarity values

\begin{tabular}{|c|c|}
\hline & $\begin{array}{c}\text { Similarity } \\
\text { value }\end{array}$ \\
\hline $\mathrm{S}\left(\mathrm{P}_{1}, \mathrm{P}_{2}\right)$ & $\mathbf{0 . 3 6}$ \\
\hline $\mathrm{S}\left(\mathrm{P}_{1}, \mathrm{P}_{3}\right)$ & $\mathbf{0 6 3}$ \\
\hline $\mathrm{S}\left(\mathrm{P}_{1}, \mathrm{P}_{4}\right)$ & $\mathbf{0 . 9 5}$ \\
\hline $\mathrm{S}\left(\mathrm{P}_{2}, \mathrm{P}_{3}\right)$ & $\mathbf{0 . 5 9}$ \\
\hline $\mathrm{S}\left(\mathrm{P}_{2}, \mathrm{P}_{4}\right)$ & $\mathbf{0 . 3 1}$ \\
\hline $\mathrm{S}\left(\mathrm{P}_{3}, \mathrm{P}_{4}\right)$ & $\mathbf{0 . 5 9}$ \\
\hline
\end{tabular}




\subsubsection{Ascending Hierarchical Classification Algorithm for (addressing) and similarity threshold}

Classifying is grouping objects together according to similar criteria. There are two main families of classification techniques:

The non-hierarchical classification or partitioning leads to the decomposition of the set of all individuals in $\mathrm{m}$ disjoint sets or equivalence classes, the number of classes is fixed for $\mathrm{m}$.

The hierarchical classification for a given accuracy, two individuals may be confused in the same group, whereas in a higher level of accuracy, they will be separated and belong to two different subgroups.

We opted for the hierarchical classification in increments of similarity that led to construct a classification tree showing the transition profiles to the group through a series of consolidation.

The obtained classification is related to the variables selected to describe individuals, in our case the developers. They are called the active variables, which will be based on the classification of individuals. For this, and to avoid dispersion of profiles similarity, the user must set the level of similarity describing each time the similarity values to consider and the level of precision represents the similarity threshold to be applied on the profiles of guidance to classify.

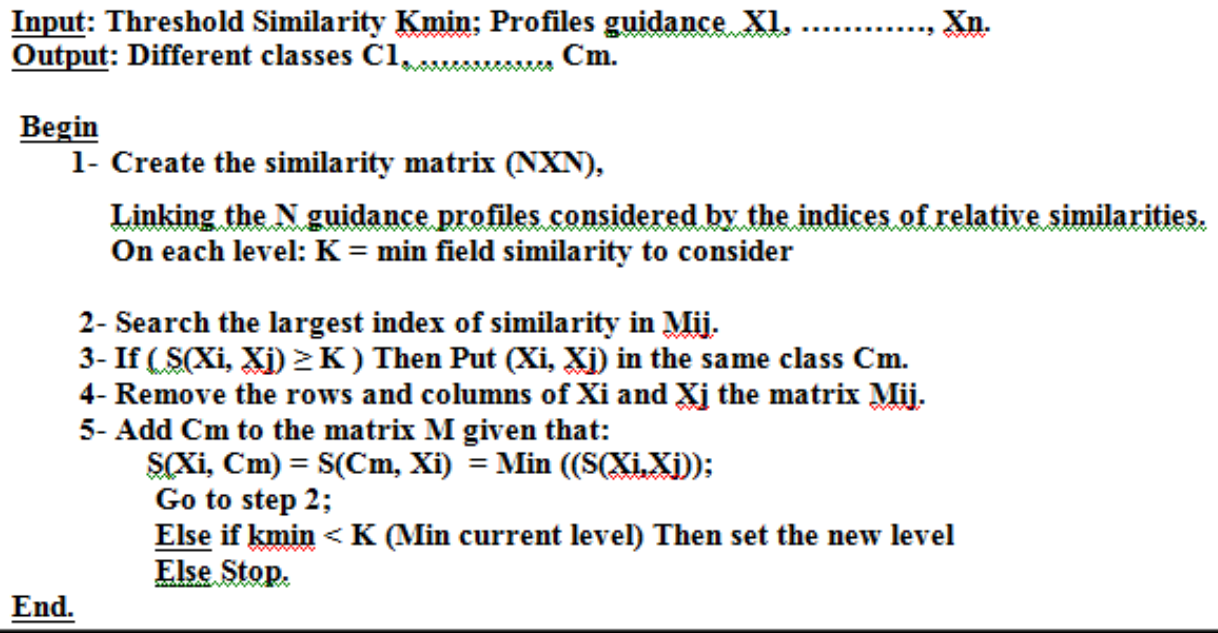

Figure 4. : Ascending Hierarchical Classification Algorithm

\subsubsection{Processing of the algorithm on a sample guidance profiles}

We have the similarity threshold set and profiles guidance $\mathrm{X} 1, \mathrm{X} 2, \ldots . . . . ., \mathrm{Xn}$ as input. Our algorithm will create a square matrix of size $(\mathrm{NXN})$ considering the number of profiles to classify and index of similarity between profiles. See the following graph of similarity: 


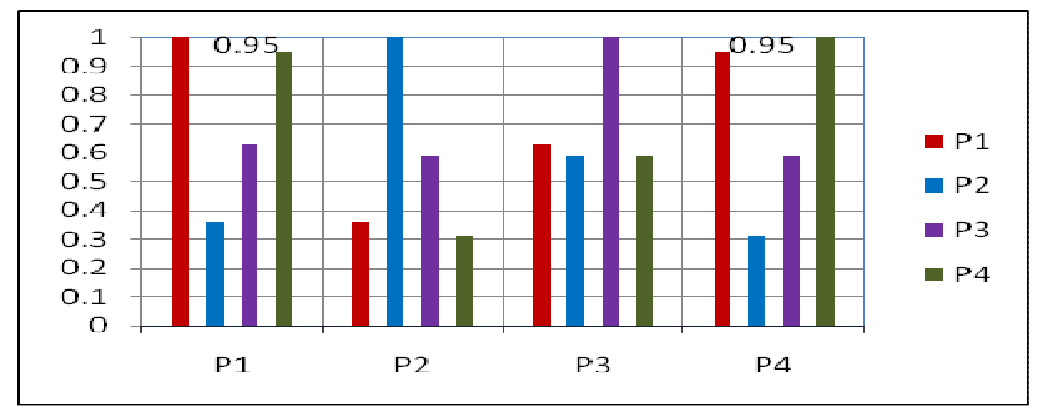

Figure 5. : The graph of similarity

For a level of 0.2 and a minimum similarity threshold of 0.50 , set initially by the user, which fixes $\mathrm{K}$ to 0.80 , the application of this algorithm is illustrated as follows:

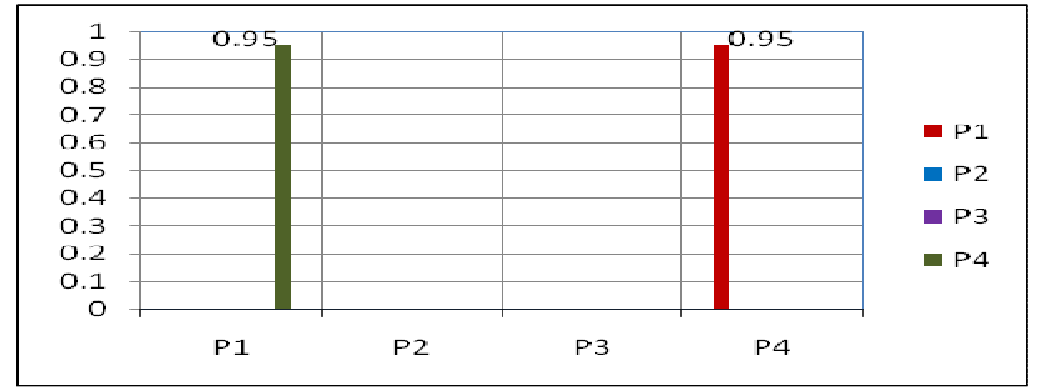

Figure 6. : Illustration of the algorithm

The maximum value of similarity in this table is 0.9 , it is the index of similarity between two profiles $\mathrm{P} 1$ and $\mathrm{P} 4$, and these profiles will be aggregated to the first group $\mathrm{C} 1$.

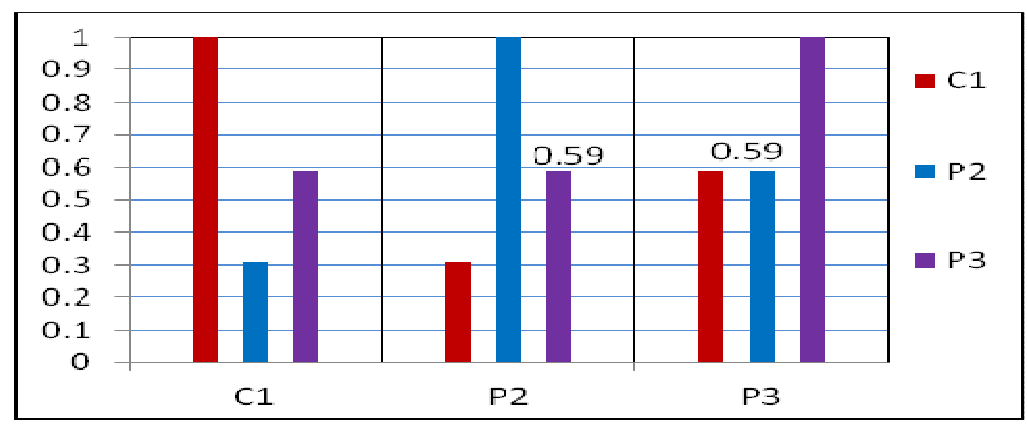

Figure 7. : Classification (first set)

At this level, we find that the similarity indices are all below the minimum level of similarity of the first set.

- Since Kmin is less than $\mathrm{K}$, we fix the next level, for this example, the new $\mathrm{K}$ will be set at 0.50 . It repeats the previous steps until all the indices similarities are below the new threshold of similarity. 
- The maximum value of similarity in the new similarity matrix is 0.59 , it is the index of similarity between two profiles $\mathrm{P} 2$ and $\mathrm{P} 3$, and the two profiles will be aggregated to the first group C2.

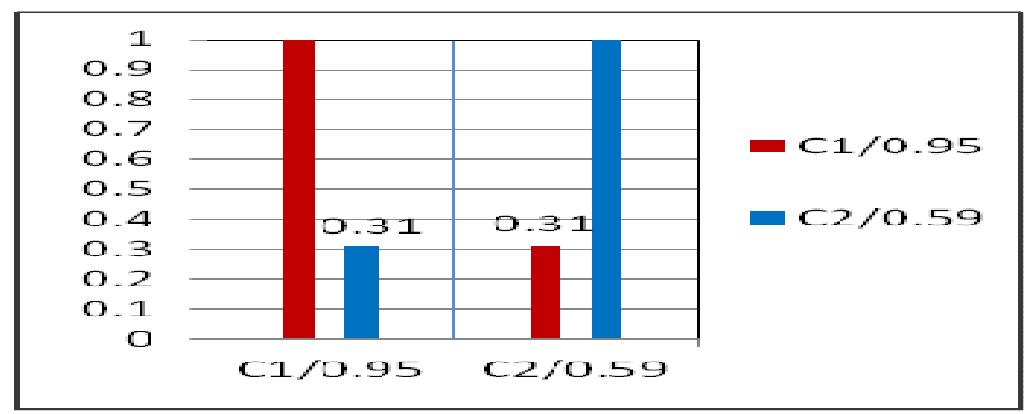

Figure 8. : The major classification

The application of our approach to similarity index and hierarchical clustering allowed us to deduce of the initial profiles number, two major classes $\mathrm{C} 1, \mathrm{C} 2$. This will provide the basis for optimal allocation of the appropriate guidance.

The guidance profile (GP) associated to each profile (Px) class is based on the following formula:

$$
G P(P x)=\sum A_{i} W_{i} / 2 * \sum W_{i} \text { avec } i=1 \text { to } 9
$$

With: $\mathrm{A}_{\mathrm{i}}$ : the characteristic value and $\mathrm{W}_{\mathrm{i}}$ : the associated weight. In our case, the guidance profile of each class based on the smallest similarity value is given by:

Table 10. The associate guidance value

\begin{tabular}{|c|c|c|}
\hline & $\mathrm{C} 1$ & $\mathrm{C} 2$ \\
\hline $\mathrm{GP}$ & $\mathbf{0 . 5 0}$ & $\mathbf{0 . 3 7}$ \\
\hline
\end{tabular}

It should be noted that the value of GP is ranged from 0 to 1 . The range associated with each type of guidance is defined by the project manager. For instance, if the range of the corrective guidance is bounded between 0 and 0.40 and the range of the constructive guidance is between 0.41 and 0.70 , we automatically associate a corrective guidance to the class $\mathrm{C} 2$ and a constructive guidance to the class $\mathrm{C} 1$.

\section{CONCLUSION}

The system presented in this paper is an approach based on similarity to process guidance model in the software process. It allows the profiles optimization, ie: classes presented through the semantics description of handled data, the definition of a system for processing the similarity index and classification of guidance profiles. The aim of this work is to facilitate the analysis of our population using the adaptive development context involved in the execution of a software process.

The system has been designed and implemented and its practical assessment seems to be promising with a significant impact on the productivity of software process development. 
In perspective and in order to improve this approach, it would be interesting to develop a similarity measure that takes into account the partial knowledge of profile characteristics. This allows the selection of a profile as the "best effort".

\section{REFERENCES}

[1] Kirk, D.C, MacDonell, S.G., \& Tempero, E. 2009 Modeling software processes - a focus on objectives, in Proceedings of the Onward, 2009. USA, ACM Press, pp.941-948.

[2] Ivan Garcia and Carla Pacheco. 2009. Toward Automated Support for Software Process Improvement Initiatives in Small and Medium Size Enterprises. Book chapter. Software Engineering Research, Management and Applications 2009. Volume 253, pp. 51-58. Springer-Verlag Berlin Heidelberg. ISBN: 978-3-642-05440-2.

[3] Hamid Khemissa, Mohamed Ahmed-Nacer, Mourad Daoudi, 2008. A Generic assistance system of software process. In proceeding of the IASTED International Conference on Software Engineering: Software Engineering. (SE '08), ACTA Press, Anaheim, CA, USA, 237-242 (C2008 February., Austria.

[4] Dadam, P. and Reichert, M. 2009. The ADEPT Project: A Decade of Research and Development for Robust and Flexible Process Support - Challenges and Achievements,' Springer, Computer Science Research and Development, 2009. Vol. 23, No. 2, pp. 81-97.

[5] MAlgOUYRES H., MOTET G. 2006. A UML Consistency Verification Approach Based on Meta modeling Formalization. Symposium on Applied Computing, Dijon, France, ACM publishers.

[6] C. EyssautierBavay, 2008. Modèles, langage et outils pour la réutilisation de profils d'apprenants", Thèse de doctorat de l'Université Joseph Fourier Grenoble 1, 26 Mai 2008.

[7] CHU09 CHUNG-FOO-WO Daniel. 2009. Adaptation dynamique par tissage d'aspects de l'optimisation. Thèse de doctorat, Université de Nice - Sophia Antipolis, 5 Mars 2009, Equipe RAINBOW, Pôle GLC.

[8] S. dami et al, 1998. APEL: a Graphical Yet Executable Formalism for Process Modeling. Kuwler Academic Publisher, pp. 60-96, Boston, January 1998.

[9] Borislava I. Simidchieva, Lori A. Clarke , Leon J. Osterweil. 2007. Representing process variation with a process family. ICSP'07 Proceedings of the 2007 international conference on Software process. Springer-Verlag Berlin, Heidelberg (C) 2007 ISBN: 978-3-540-72425-4.

[10] Coulette Bernard., Crégut Xavier. et al, 2000. RHODES, a Process-centered Software Engineering Environment, in Proc. of ICEIS 2000, Stafford, pp 253-260, 2000.

[11] Bradshaw J., Editor,. 2000. Handbook of Agent Technology. MIT Press, 2000.

[12] OMG. Inc. 2008. Software Engineering Meta-Model Specification version 2.0: Formal/2008-04- 01.

[13] Vivien Robinet, Gilles Bisson, Mirta B. Gordon, Benoît Lemaire. 2007. Induction of High-level Behaviors from Problem-solving Traces using Machine Learning Tools. Published in "IEEE Intelligent Systems 22, 4 (2007) 22".

[14] Ahmed Belkhirat, Abdelghani Bouras, Abdelkader Belkhir. 2009. A New Similarity Measure for the Anomaly Intrusion Detection. In Third International Conference on Network and System Security (NSS).

[15] Ahmed Belkhirat, Abdelkader Belkhir, Abdelghani Bouras 2011 A New Similarity Measure for the Profiles Management, UKSIM '11: Proceedings of the Tenth International Conference on Computer Modeling and Simulation, Cambridge England.

[16] Xavier Aimé, Frédéric Furst, Pascale Kuntz, Francky Trichet. 2009. «SEMIOSEM: A Semiotic-Based Similarity Measure". On the Move to Meaningful Internet Systems (OTM 2009), International Workshop on Ontology content and evaluation in Enterprise (OntoContent'2009), Lecture Notes in Computer Science-LNCS 5872, pp. 584-593. Springer-Verlag (Berlin Heidelberg). ISBN 978-3-64205289-7. Villamoura, Portugal.

[17] S. Boriah, V. Chandola, and V. Kumar. 2008. Similarity measures for categorical data: A comparative evaluation. In SDM 2008: Proceedings of the eighth SIAM International Conference on Data Mining, pages 243-254, 2008.

[18] Fernando Lourenco, Victor Lobo, Fernando 2004. Bacao: Binary-based similarity measures for categorical data and their application in self-organizing maps. JOCLAD - XI Days of classification and analysis of data, April 1-3, Lisbon. 
[19] Barry W. Boehm, Chris Abts, A. Winsor Brown, Sunita Chulani, Bradford K. Clark, Ellis Horowitz, Ray Madachy, Donald J. Reifer, Bert Steece. 2009 Software Cost Estimation With COCOMO II. Prentice Hall Edition, ISBN: 0137025769, 978013702576.

[20] Kirk, D., \& MacDonell, S. 2009. A simulation framework to support software project (re)planning, in Proceedings of the 35th Euromicro Software Engineering and Advanced Applications (SEAA) Conference. Patras, Greece, IEEE Computer Society Press, pp.285-292. 